\title{
Ferromagnetism and Superconductivity in the multi-orbital Hubbard Model: Hund's Rule Coupling versus Crystal-Field Splitting
}

\author{
Kazuhiro SANO* and Yoshiaki ŌNO 1 \\ Department of Physics Engineering, Mie University, Tsu, Mie 514-8507 \\ 1 Department of Physics, Nagoya University, Nagoya 464-8602
}

(Received October 8, 2018)

\begin{abstract}
The multi-orbital Hubbard model in one dimension is studied using the numerical diagonalization method. Due to the effect of the crystal-field splitting $\Delta$, the fully polarized ferromagnetism which is observed in the strong coupling regime becomes unstable against the partially polarized ferromagnetism when the Hund's rule coupling $J$ is smaller than a certain critical value of order of $\Delta$. In the vicinity of the partially polarized ferromagnetism, the orbital fluctuation develops due to the competition between the Hund's rule coupling and the crystal-field splitting. The superconducting phase with the Luttinger liquid parameter $K_{\rho}>1$ is observed for the singlet ground state in this region.
\end{abstract}

KEYWORDS: electronic structure, ferromagnetism, superconductivity, Luttinger liquid, Hubbard model

Recently, the strongly correlated electron systems with orbital degrees of freedom have attracted much interest. Striking phenomena of such systems are the colossal magnetoresistance in manganites $\mathrm{La}_{1-x} \mathrm{Sr}_{x} \mathrm{MnO}_{3},{ }^{1)}$ the triplet pairing superconductivity in the ruthenate $\mathrm{Sr}_{2} \mathrm{RuO}_{4}{ }^{2)}$ and the metal-insulator transition in alkalidoped fullerides $\mathrm{A}_{x} \mathrm{C}_{60} \cdot{ }^{3)}$

Another interesting class of materials is cobalt oxides such as $\mathrm{La}_{1-x} \mathrm{Sr}_{x} \mathrm{CoO}_{3}{ }^{4-6)}$ which shows the spin state transition. Because the Hund's rule coupling and the crystal-field splitting between the $t_{2 g}$ and $e_{g}$ orbitals are close to each other, the spin state of the cobalt ion depends on temperature, doping concentration and crystal structure. For example, the ground state of the $\mathrm{Co}^{3+}$ $\left(3 d^{6}\right)$ ion in $\mathrm{LaCoO}_{3}$ is known to be a low-spin (LS) state $\left(t_{2 g}^{6} e_{g}^{0}, S=0\right)$, while, with increasing temperature, the spin state gradually changes into an intermediatespin (IS) state $\left(t_{2 g}^{5} e_{g}^{1}, S=1\right)$ and/or a high-spin (HS) state $\left(t_{2 g}^{4} e_{g}^{2}, S=2\right) .{ }^{5-8)}$ With $\mathrm{Sr}$ doping, $\mathrm{La}_{1-x} \mathrm{Sr}_{x} \mathrm{CoO}_{3}$ shows a spin-glass for $x<0.18$ and a ferromagnetism for $x>0.18,{ }^{9)}$ where IS and/or HS states play crucial roles.

The recent discovery of large thermoelectric power in $\mathrm{Na}_{0.5} \mathrm{CoO}_{2}{ }^{10)}$ has stimulated considerable attention on layered cobalt oxides such as $\mathrm{Na}_{x} \mathrm{CoO}_{2}$. Koshibae et al. ${ }^{11)}$ claimed that large degeneracy of electronic states due to a competition between the Hund's rule coupling and the crystal-field splitting, together with strong correlation plays a key role in $\mathrm{Na}_{x} \mathrm{CoO}_{2}$ as well as $\mathrm{La}_{1-x} \mathrm{Sr}_{x} \mathrm{CoO}_{3}$. Weak ferromagnetism has been observed in $\mathrm{Na}_{0.75} \mathrm{CoO}_{2} .{ }^{12)}$ More recently, Takada et al. ${ }^{13)}$ have discovered the superconductivity in $\mathrm{Na}_{x} \mathrm{CoO}_{2}$. $y \mathrm{H}_{2} \mathrm{O}$ with $T_{c} \approx 5 K$ for $x \approx 0.35$ and $y \approx 1.3$. With the advent of the new findings, theoretical studies of the interplay of the Hund's rule coupling and the crystal-field splitting is highly desirable.

The orbitally degenerate Hubbard model has been extensively investigated to clarify the effect of orbital degrees of freedom in the presence of the intra-atomic Coulomb interaction. Many authors ${ }^{14-19)}$ have studied the ferromagnetism of this model and revealed that the Hund's rule coupling plays a crucial role in the ferromagnetism; however the effect of the crystal-field splitting was not considered there. Possible mechanisms of superconductivity have been proposed by several authors, ${ }^{20-23)}$ but the relationship with the ferromagnetism was not discussed there.

In the present work, we investigate the ferromagnetism and the superconductivity in the multi-orbital Hubbard model, in particular paying attention to the effect of the interplay of the Hund's rule coupling $J$ and the crystalfield splitting $\Delta$. As the strong correlation effect plays crucial roles in ferromagnetism, a non-perturbative and reliable approach is required. We employ the numerical diagonalization method for the one-dimensional Hubbard model with finite system sizes. This approach has already been applied for the $\Delta=0$ case. ${ }^{14,15)}$ Although the available system size is fairly small, the results are in good agreement with the strong coupling analysis ${ }^{15}$ ) and the results from the density-matrix renormalizationgroup method. ${ }^{19)}$ To examine the superconductivity, we calculate the critical exponent of the correlation functions $K_{\rho}$ based on the Luttinger liquid theory. ${ }^{24,25)}$ The reliability of this approach has been extensively tested for various one-dimensional models such as the Hubbard model,${ }^{26)}$ the $t$ - $J$ model, ${ }^{27)}$ the $d-p$ model,${ }^{28-30)}$ etc. We can thus expect that this approach is reliable for multiorbital Hubbard model as well.

We consider the following Hamiltonian for the onedimensional multi-orbital Hubbard model:

$$
\begin{aligned}
H & =-t \sum_{i, m, \sigma}\left(c_{i, m, \sigma}^{\dagger} c_{i+1, m, \sigma}+\text { h.c. }\right) \\
& +U \sum_{i, m} n_{i, m, \uparrow} n_{i, m, \downarrow}+U^{\prime} \sum_{i, \sigma} n_{i, a, \sigma} n_{i, b,-\sigma} \\
& +\left(U^{\prime}-J\right) \sum_{i, \sigma} n_{i, a, \sigma} n_{i, b, \sigma}+\frac{\Delta}{2} \sum_{i, \sigma}\left(n_{i, a, \sigma}-n_{i, b, \sigma}\right)
\end{aligned}
$$




$$
\begin{aligned}
& -J \sum_{i, m, \sigma}\left(c_{i, a, \uparrow}^{\dagger} c_{i, a, \downarrow} c_{i, b, \downarrow}^{\dagger} c_{i, b, \uparrow}+\text { h.c. }\right) \\
& -J^{\prime} \sum_{i, m, \sigma}\left(c_{i, a, \uparrow}^{\dagger} c_{i, a, \downarrow}^{\dagger} c_{i, b, \uparrow} c_{i, b, \downarrow}+\text { h.c. }\right)
\end{aligned}
$$

where $c_{i, m, \sigma}^{\dagger}$ stands for creation operator of a electron with spin $\sigma$ in the orbital $m(=a, b)$ at site $i$ and $n_{i, m, \sigma}=c_{i, m, \sigma}^{\dagger} c_{i, m, \sigma}$. Here, $t$ represents the hopping integral between the same orbitals and we set $t=1$ in this study. The interaction parameters $U, U^{\prime}, J$ and $J^{\prime}$ stand the intra- and inter-orbital direct Coulomb interactions, the exchange (Hund's rule) coupling and the pairtransfer, respectively. $\Delta$ stands the energy difference between the two atomic orbitals, i.e., the crystal-field splitting. For simplicity, we impose the relations, $J=J^{\prime}$ and $U=U^{\prime}+2 J$, which holds exactly in $3 d$-orbitals for $\Delta=0$ and is a good approximation for $\Delta \neq 0$. The model eq.(1) is schematically represented by Fig.1(a).

To carry out a systematic calculation, we use the periodic boundary condition for $N_{e}=4 m+2$ and the antiperiodic boundary condition for $N_{e}=4 m$, where $N_{e}$ is the total electron number and $m$ is an integer. This choice of the boundary condition removes accidental degeneracy so that the ground state might always be a singlet with zero momentum except for the ferromagnetic state at large $J$. The filling $n$ is defined by $n=N_{e} / N_{u}$, where $N_{u}$ is the total number of unit cells (each unit cell contains two orbitals). We numerically diagonalize the Hamiltonian eq.(1) up to 14 sites ( 7 unit cells) using the standard Lanczos algorithm.

When the charge gap vanishes in the thermodynamic limit, the uniform charge susceptibility $\chi_{c}$ is obtained from $\chi_{c}=\frac{4 / N_{u}}{E_{0}\left(N_{e}+2, N_{u}\right)+E_{0}\left(N_{e}-2, N_{u}\right)-2 E_{0}\left(N_{e}, N_{u}\right)}$, where $E_{0}\left(N_{e}, N_{u}\right)$ is the ground state energy of a system with $N_{u}$ unit cells and $N_{e}$ electrons. In the Luttinger liquid theory, some relations have been established as universal relations in one-dimensional models. ${ }^{24,25)}$ In the model which is isotropic in spin space, the critical exponents of various types of correlation functions are determined by a single parameter $K_{\rho}$. It is predicted that the superconducting (SC) correlation is dominant for $K_{\rho}>1$ (the correlation function decays as $\sim r^{-\left(1+\frac{1}{K_{\rho}}\right)}$ ), whereas the CDW or SDW correlations are dominant for $K_{\rho}<1$ (the correlation functions decay as $\sim r^{-\left(1+K_{\rho}\right)}$ ) in the Tomonaga-Luttinger regime. ${ }^{25)}$ The critical exponent $K_{\rho}$ is related to the charge susceptibility $\chi_{c}$ and the Drude weight $D$ by $K_{\rho}=\frac{1}{2}\left(\pi \chi_{c} D\right)^{1 / 2}$, with $D=\frac{\pi}{N_{u}} \frac{\partial^{2} E_{0}(\phi)}{\partial \phi^{2}}$, where $E_{0}(\phi)$ is the total energy of the ground state as a function of a magnetic flux $N_{u} \phi{ }^{25)}$

In the noninteracting case $\left(U=U^{\prime}=J=0\right)$, the Hamiltonian eq.(1) yields a dispersion relation $\epsilon^{ \pm}(k)=$ $-2 t \cos (k) \pm \frac{\Delta}{2}$, where $k$ is the wave vector and $\epsilon^{+}(k)$ $\left(\epsilon^{-}(k)\right)$ represents the upper (lower) band energy. When the lowest energy of the upper band, $\epsilon^{+}(0)$, is larger than the Fermi energy $E_{k_{F}}$, electrons occupy only the lower band with $k_{F}=\frac{\pi n}{2}$. This band structure is schematically represented by Fig.1(b). Hereafter, we mainly consider the case with $\epsilon^{+}(0)>E_{k_{F}}$.

Figure 2 shows the value of $K_{\rho}$ as a function of $J\left(=U^{\prime}\right)$

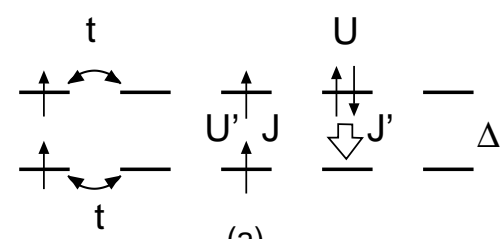

(a)

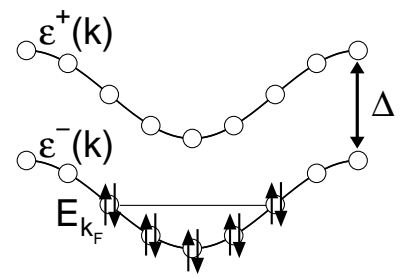

(b)

Fig. 1. Schematic diagrams of (a) the model Hamiltonian and (b) the band structure in the noninteracting case.

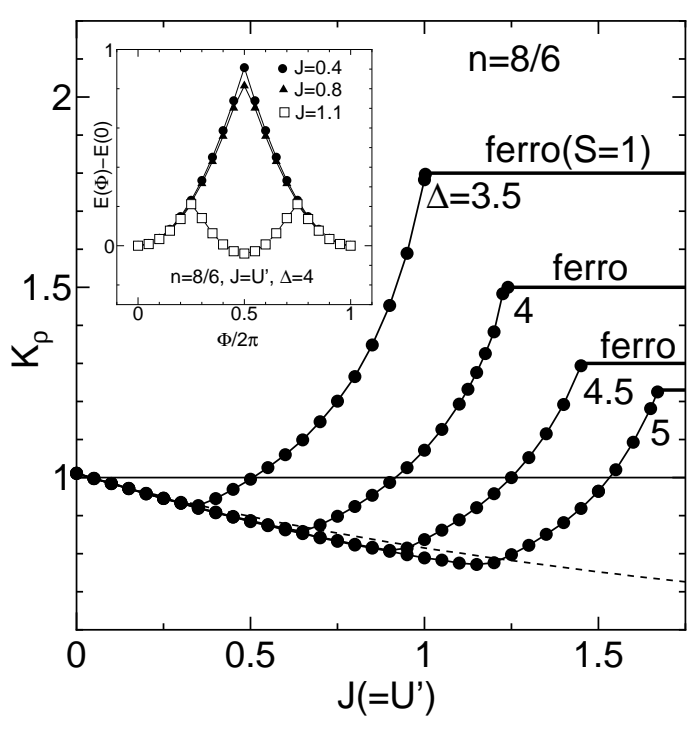

Fig. 2. $K_{\rho}$ as a function of $J\left(=U^{\prime}\right)$ at $\Delta=3.5,4,4.5$ and 5 . The broken line represents a weak coupling estimation for $K_{\rho}$. Inset shows the energy difference $E_{0}(\phi)-E_{0}(0)$ as a function of an external flux $\phi$.

for several values of $\Delta$ at the electron density $n=8 / 6$. As $J$ increases $K_{\rho}$ decreases for small $J$, while it increases for large $J$ and, then, becomes larger than unity. In the region $K_{\rho}>1$, the $\mathrm{SC}$ correlation is expected to be most dominant compared to the CDW and SDW correlations. When $J$ is larger than a certain critical value, the ground state changes into the partially ferromagnetic state with $\mathrm{S}=1$ from the singlet state.

In Fig. 2 the broken line represents a weak coupling estimation for $K_{\rho}$, where $E_{0}$ is calculated within the first order perturbation (Hartree approximation). ${ }^{31,32)}$ This approximation shows a good agreement with the numerical result in the weak coupling regime, ensuring the small finite-size effect of the numerical calculation. In this approximation, the ground state energy is obtained by the expectation value of the Hamiltonian eq.(1) using the noninteracting ground state where the lower band is exclusively occupied as shown in Fig.1(b). Then the effect 


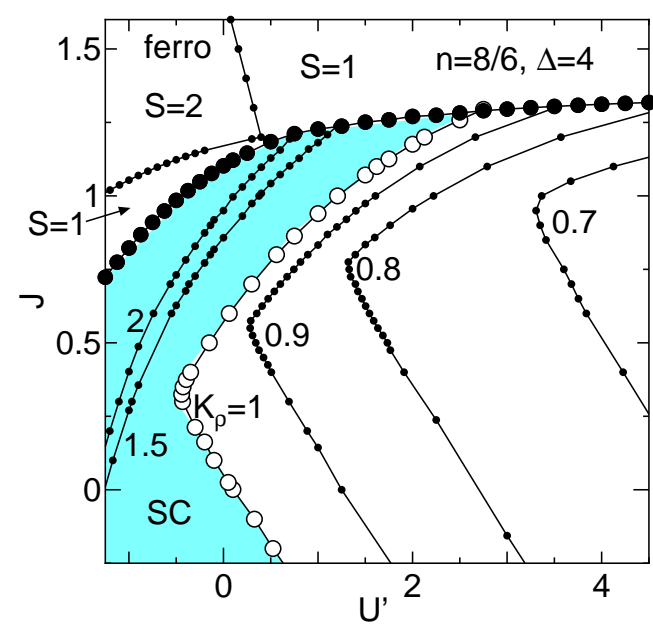

Fig. 3. Phase diagram of the superconducting state with $K_{\rho}>1$ (shadowed region) and the ferromagnetic state with $S \neq 0$ on the $U^{\prime}-J$ parameter plane with contour map of $K_{\rho}$.

of the upper band is omitted and the result is independent of $\Delta$ as shown in Fig.2. This approximation breaks down when the effect of the upper band becomes crucial. In this regime, $K_{\rho}$ rapidly increases with increasing $J$, and finally becomes larger than unity showing the superconducting state.

The critical values of $J$ with $K_{\rho}=1$ are $J_{c} \approx 0.5$, $0.9,1.3$ and 1.6 for $\Delta=3.5,4,4.5$ and 5 , respectively. We find that the phenomenological equation $J_{c}=\epsilon^{+}(0)-E_{k_{F}}$ can approximately lead to the above results. Here, $\epsilon^{+}(0)-E_{k_{F}}$ corresponds to the lowest energy of the single-particle excitation from the lower band to the upper band. This equation is found to be a good approximation for various filling $n$. When $J$ exceeds $\epsilon^{+}(0)-E_{k_{F}}$, the inter-band excitation develops and the occupation number of the upper band increases, which results in the large orbital fluctuation accompanied by the fluctuation between the low-spin and the high-spin states. The mechanism of the superconductivity is related to this orbital fluctuation.

We note that the superconductivity is also observed for the $J^{\prime}=0(J \neq 0)$ case in contrast to the previous study $^{21,22)}$ where the pair-transfer $J^{\prime}$ is crucial for the superconductivity. The importance of the upper band has also been pointed out in different types of multi-band models such as the $d-p \operatorname{model}^{28-30,33)}$ and the Hubbard ladder model. ${ }^{31,32)}$

To confirm the superconducting state, we calculate the lowest energy of the singlet state $E_{0}(\phi)$ as a function of an external flux $\phi$. As shown in the inset of Fig.2, the anomalous flux quantization occurs at $J=1.1$, where $K_{\rho}$ is about 1.4. When $J=0.8$ and $0.4, K_{\rho}$ is less than unity and the anomalous flux quantization is not found.

In Fig.3, we show the phase diagram of the superconducting state with $K_{\rho}>1$ together with the ferromagnetic state with $S \neq 0$ on the $U^{\prime}$ vs. $J$ parameter plane, where the value of $K_{\rho}$ is given by the contour map. The superconducting phase appears near the partially polarized ferromagnetic region. It extends from the attractive region with $J<0$ and $U^{\prime}<0$ to the realistic param-

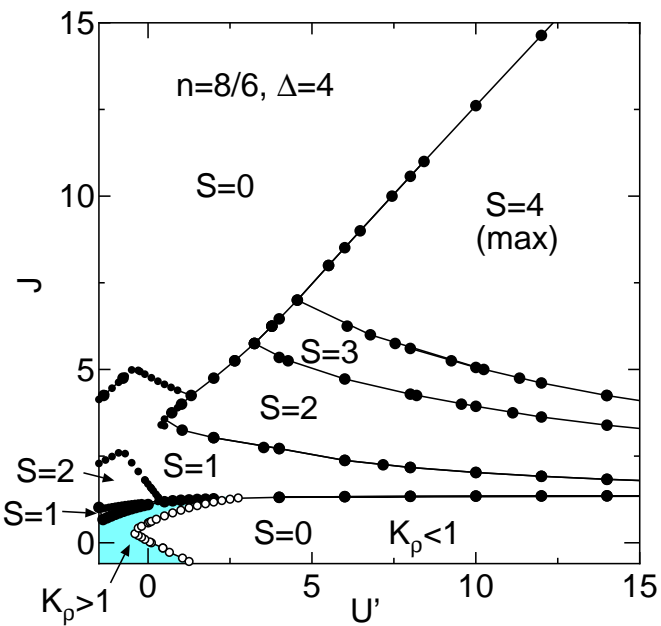

Fig. 4. Global phase diagram on the $U^{\prime}-J$ plane.

eter region for $3 d$ transition-metals with $U^{\prime}>J>0$. We have confirmed that the superconducting region increases as $\Delta$ decreases as shown in Fig.2.

Fig.4 shows the global phase diagram on the $U^{\prime}-J$ plane. When $U^{\prime} \gtrsim J \gtrsim \Delta$, the fully polarized ferromagnetism with $S=S_{\max }$ appears. It accompanies the partially polarized ferromagnetism with $0<S<S_{\max }$ for $J \lesssim \Delta$. In the $\Delta=0$ case, the ferromagnetism of the degenerate Hubbard model has been previously studied. ${ }^{14-19)}$ In one dimension, some rigorous results are shown in the strong coupling limit $U \rightarrow \infty$ : the ground state is fully polarized ferromagnetism for $0<n<2$ except for $n=1$ when $U^{\prime}$ and $J=J^{\prime}$ are positive and finite. ${ }^{17)}$ Numerical result suggests that the ferromagnetism is stable also for $n=1$ in the strong coupling region. ${ }^{19)}$ In infinite dimensions, the dynamical meanfield theory shows the existence of the ferromagnetism in the same parameter region observed in one dimension. ${ }^{18)}$ The ferromagnetism for $n \neq 1$ is found to be metallic and mainly caused by the double-exchange mechanism, ${ }^{19)}$ which is also the present case with $\Delta \neq 0$. The existence of the partially polarized ferromagnetism for $\Delta>0$ has been reported in a different type of the twoband Hubbard model. ${ }^{34)}$ The ferromagnetic phase for $U^{\prime} \lesssim 0$ is complicated as shown in Fig.4. The origin of this phase is not clear at this stage.

In Fig.5, we show the doping dependence of the critical values of $J$ for the superconductivity and the ferromagnetism at $\Delta=4$ with $J=U^{\prime}$, where we use $n=8 / 6,10 / 7$, $6 / 4,8 / 5$ and $12 / 7$ systems. The critical values are defined above which $K_{\rho}>1$ for the superconductivity and $S>0$ for the ferromagnetism, respectively. Although the finite size effect is considerably large, the phase boundary for the superconductivity can be approximately given by the phenomenological equation, $J_{c}=\epsilon^{+}(0)-E_{k_{F}}$, mentioned before.

For $n=8 / 7$ and $n=6 / 5$, we cannot use the expression $K_{\rho}=\frac{1}{2}\left(\pi \chi_{c} D\right)^{1 / 2}$, because the system has a charge gap at $N_{e}=N_{u}$ and, then, $\chi_{c}$ is not obtained from the finite size systems. Instead we use another expression, $K_{\rho}=\frac{D}{2 v_{c}}$ with the charge velocity $v_{c}$. Although there is a larger finite size effect, we find $K_{\rho}<1$ until the 


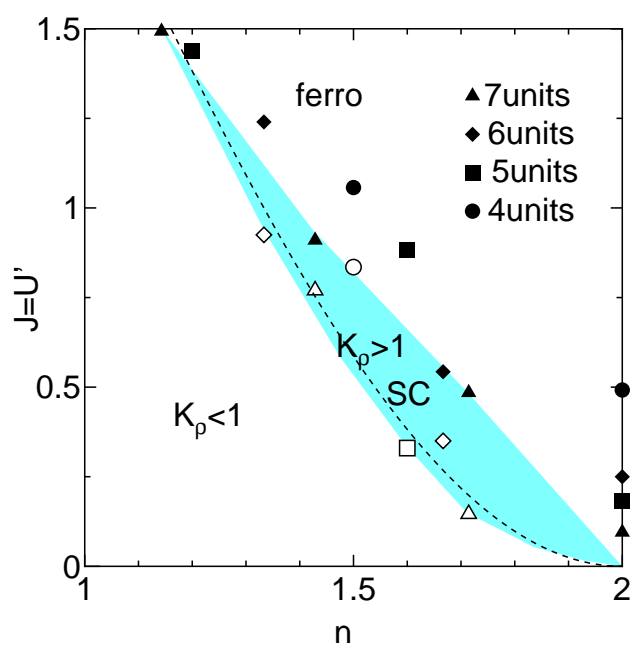

Fig. 5. Phase diagram on the $n-J$ plane. The broken line represents the phenomenological equation $J_{c}=\epsilon^{+}(0)-E_{k_{F}}$ (see text) as a function of $n$.

ferromagnetic transition occurs. Furthermore, the result of the flux quantization also shows no sign of the superconductivity in this case. These results suggest that the superconducting phase may disappear for $n \lesssim 6 / 5$.

In conclusion, we have obtained the phase diagram of the one-dimensional Hubbard model with two-fold orbital degeneracy. The fully polarized ferromagnetism has been found in the strong coupling regime with $U^{\prime} \gtrsim$ $J \gtrsim \Delta$. For $1<n<2$, the ferromagnetism is metallic and mainly caused by the double-exchange mechanism. ${ }^{19)}$ The crystal-field splitting destroys the fully polarized ferromagnetism resulting in the partially polarized one for $J \lesssim \Delta$. In the vicinity of the partially polarized ferromagnetism, we have found the superconducting phase, when $J$ exceeds the lowest energy of the inter-band excitation, which extends to the realistic parameter region for $3 d$ transition-metals with $U^{\prime}>J$.

Sakamoto et al. ${ }^{19)}$ claimed that the metallic ferromagnetism appears in a similar parameter region in any dimension by comparing the results from one dimension with those from infinite dimensions. It is natural to think that this ferromagnetism will appear in two and three dimensions even in the presence of $\Delta$. Then, we expect that a partially polarized (weak) ferromagnetism appears in real materials, in which the Hund's rule coupling and the crystal-field splitting compete to each other, such as cobalt oxides. In fact a weak ferromagnetism has been observed in the layered $\mathrm{Na}_{0.75} \mathrm{CoO}_{2}{ }^{12)}$ as well as the perovskite $\mathrm{R}_{1-x} \mathrm{~A}_{x} \mathrm{CoO}_{3} .{ }^{39)}$

Finally we make some comments on the superconductivity in $\mathrm{Na}_{x} \mathrm{CoO}_{2} \cdot y \mathrm{H}_{2} \mathrm{O} .{ }^{13)}$ Although the several authors $^{35-38)}$ have discussed the system using the nondegenerate $t$ - $J$ model, the orbital degeneracy of $3 d$ electrons is considered to play a crucial role in $\mathrm{Na}_{x} \mathrm{CoO}_{2}$ as well as $\mathrm{La}_{1-x} \mathrm{Sr}_{x} \mathrm{CoO}_{3} .{ }^{11)}$ The competition between the Hund's rule coupling and the crystal-field splitting causes the large orbital fluctuation, accompanied by the fluctuation between the low-spin and the high-spin states at each Co ion, which mediates the superconductivity. As the orbital fluctuation has a local character, the mech- anism for the superconductivity could be common in all dimensions. We hence expect that the multi-orbital mechanism is responsible for the superconductivity in layered $\mathrm{Na}_{x} \mathrm{CoO}_{2} \cdot y \mathrm{H}_{2} \mathrm{O}$. Exploration of the superconductivity in the vicinity of the weak ferromagnetism in the perovskite $\mathrm{R}_{1-x} \mathrm{~A}_{x} \mathrm{CoO}_{3}{ }^{39)}$ may be promising.

We would like to thank Prof. Masatoshi Sato who led our attention to cobalt oxides and suggested the importance of the Hund's rule coupling on the superconductivity. This work was partially supported by the Grantin-Aid for Scientific Research from the Ministry of Education, Science, Sports and Culture.

1) For a review, see, for example, Y. Tokura, Ed.: Colossal Magnetoresistive Oxides (Gordon and Breach Science, New York, 2000).

2) Y. Maeno, H. Hashimoto, K. Yoshida, S Nishizaki, T. Fujita, J. G. Bednorz and F. Lichtenberg: Nature (London) 372 (1994) 532 .

3) O. Gunnarsson, E. Koch and R. M. Martin: Phys. Rev. B 54 (1996) R11026.

4) G. H. Jonker and J. H. Van Santen: Physica 19 (1953) 120.

5) R. R. Heikes, R. C. Miller and R. Mazelsky: Physica 30 (1964) 1600.

6) P. M. Raccah and J. B. Goodenough: Phys. Rev. 155 (1967) 932.

7) K. Asai, P. Gehring, H. Chou, and G. Shirane: Phys. Rev. B 40 (1989) 10982.

8) M. Itoh, M. Sugahara, I. Natori and K. Motoya: J. Phys. Soc. Jan. 64 (1995) 3967.

9) D. Louca, J. L. Sarrao, J. D. Thompson, H. Roder and G. H. Kwei: Phys. Rev. B 60 (1999) 10378.

10) I. Terasaki, Y. Sasago and K. Uchinokura, Phys. Rev. B 56 (1997) R12685.

11) W. Koshibae, K. Tsutsui and S. Maekawa, Phys. Rev. B 62 (2000) 6869.

12) T. Motohashi, R. Ueda, E. Naujalis, T. Tojo, I. Terasaki, T. Atake, M. Karppinen and H. Yamauchi: Phys. Rev. B 67 (2003) 064406.

13) K. Takada, H. Sakurai, E. Takayama-Muromachi, F. Izumi, R. A. Dilanian and T. Sasaki: Nature 422 (2003) 53.

14) W. Gill and D. J. Scalapino: Phys. Rev. B 35 (1987) 215.

15) J. Kuei and R. T. Scalettar: Phys. Rev. B 55 (1997) 14968.

16) K. Held and D. Vollhardt: Eur. Phys. J. B 5 (1998) 473.

17) S. Q. Shen: Phys. Rev. B57 (1998) 6474.

18) T. Momoi and K. Kubo: Phys. Rev. B 58 (1998) R567.

19) H. Sakamoto, T. Momoi and K. Kubo: Phys. Rev. B 65 (2002) 224403.

20) T. Takimoto: Phys. Rev. B 62 (2000) R14641.

21) M. Imada: J. Phys. Soc. Jpn. 70 (2001) 1218.

22) K. Yamaji: J. Phys. Soc. Jpn. 70 (2001) 1476.

23) T. Nomura and K. Yamada: J. Phys. Soc. Jan. 71 (2002) 1993.

24) F.D.M. Haldane: J. Phys. C14 (1981) 2585.

25) J. Voit: Rep. Prog. Phys. 58 (1995) 977.

26) H. J. Schulz: Phys. Rev. Lett. 64 (1990) 2831.

27) M. Ogata, M. U. Luchini, S. Sorella and F. F. Assaad: Phys. Rev. Lett. 66 (1991) 2388.

28) K. Sano and Y. Ōno: Physica C205 (1993) 170.

29) K. Sano and Y. Ōno: Phys. Rev. B51 (1995) 1175.

30) K. Sano and Y. Ōno: Physica C242 (1995) 113.

31) K. Sano: Physica B281\&282 (2000) 829.

32) K. Sano: J. Phys. Soc. Jpn. 69 (2000) 1000.

33) Y. Ōno and K. Sano: J. Phys. Soc. Jpn. Suppl. 71 (2002) 356.

34) K. Kusakabe, S. Watanabe and Y. Kuramoto: J. Phys. Soc. Jpn. Suppl. 71 (2002) 311.

35) G. Baskaran: cond-mat/0303649.

36) Brijesh Kumar and B. Sriram Shastry: cond-mat/0304210.

37) Q.-H. Wang, D.-H. Lee and P. A. Lee: cond-mat/0304377.

38) M. Ogata: cond-mat/0304405.

39) H. Masuda, T. Fujita, T. Miyashita, M. Soda, Y. Yasui, Y. Kobayashi and M. Sato: J. Phys. Soc. Jpn. 72 (2003) 873. 